\title{
Glutamate Neurotoxicity in vitro: Antagonist Pharmacology and Intracellular Calcium Concentrations
}

\author{
Robin L. Michaels ${ }^{1}$ and Steven M. Rothman ${ }^{2}$ \\ 'Department of Biological Chemistry and 'Departments of Pediatrics, Neurology, and Anatomy and Neurobiology, \\ Washington University School of Medicine, St. Louis, Missouri 63110
}

\begin{abstract}
There is now convincing evidence that excessive accumulation of the excitatory amino acid glutamate (GLU) in the extracellular space is toxic to central mammalian neurons. However, the role of different GLU receptors in producing this toxicity has not been adequately ascertained. There is also no adequate information about the correlation of free intracellular calcium concentration with eventual excitotoxic death. We have used cultured rat hippocampal neurons to address these issues. Approximately $75 \%$ of our neurons died after a 20-min GLU exposure. The potent kainate/quisqualate receptor antagonist 6-cyano-7-nitroquinoxaline-2,3dione did not significantly ameliorate the GLU toxicity, while the selective noncompetitive $\mathrm{N}$-methyl-D-aspartate (NMDA) antagonist methyl-10,11-dihydro-5-H-dibenzocyclohepten5,10-imine (MK-801) blocked the GLU toxicity for periods of at least $2 \mathrm{hr}$. Interestingly, kainate was very toxic to the hippocampal neurons, but this toxicity was markedly attenuated by MK-801. These results suggest that the major toxicity of GLU is mediated by NMDA receptors and that under some conditions kainate toxicity reflects nonspecific opening of NMDA channels. The intracellular calcium concentrations in these neurons at the end of exposure to GLU and kainate (in the presence and absence of different antagonists) correlated poorly with eventual survival. Antagonists that limited the rise in calcium were still ineffective in preventing death. These results confirm earlier observations that stressed the importance of NMDA receptors in mediating GLU toxicity. However, they indicate that the relationship between toxicity and neuronal calcium concentration may be very complicated. An unexpected finding of these experiments was that MK-801, unlike competitive antagonists of GLU, elevated intracellular calcium.
\end{abstract}

Over the past decade interest in the neurobiology of glutamate (GLU) has greatly increased. Part of this interest stems from the recognition of GLU as the major fast excitatory neurotransmitter in the mammalian central nervous system (Mayer and

\footnotetext{
Received May 22, 1989; revised July 10, 1989; accepted July 13, 1989.

We thank Dr. Elliot Elson for allowing us to use his laboratory for the calcium determinations, Nancy Lancaster for technical help, and Deborah Howard for typing the manuscript. This work was supported by N.I.H. grants NS19988 (S.M.R.) and GM38838 (Elliot Elson), American Heart Association grant 861413 (R.L.M.), and by the Monsanto Corporation.

Correspondence should be addressed to Steven M. Rothman, M.D., Department of Anatomy and Neurobiology, Washington University School of Medicine, St. Louis, MO 63110.

Copyright (C) 1990 Society for Neuroscience $0270-6474 / 90 / 010283-10 \$ 02.00 / 0$
}

Westbrook, 1987). Additional attention is due to recent observations that endogenous GLU is toxic to neurons when it accumulates in the extracellular space in the brain. This occurs under conditions of hypoxia and ischemia (Rothman and Olney, 1986) and experimental focal epilepsy (Collins and Olney, 1982). Because of this possible connection with naturally occurring neurological disease, defining the mechanism(s) of GLU neurotoxicity has become an important endeavor in neurobiology.

Early work on excitotoxicity emphasized the importance of kainate (KA) and its receptor in producing this type of neuronal damage (Coyle, 1983). More recently, Choi and his colleagues (1988) and Finkbeiner and Stevens (1988) have shown that specific blockade of the $N$-methyl-D-aspartate (NMDA) receptor largely blocks the toxicity of exogenous GLU in cultured cortical neurons. In addition, they and others (Simon et al., 1984; Garthwaite et al., 1986; Choi, 1987) have stressed the rolc of calcium in mediating this type of cell death.

However, a number of questions still remain unanswered. It is not known whether blockade of the NMDA receptor or NMDA-gated channel is necessary to prevent GLU toxicity or just sufficient. It is possible that blocking the non-NMDA receptors that bind GLU [i.e., KA and quisqualate (QUIS) receptors] might also reduce GLU toxicity. In addition, previous studies examined toxicity after a brief ( $5 \mathrm{~min}$ ) GLU exposure (Choi et al., 1987, 1988). With long GLU exposures NMDA blockade might be ineffective in limiting GLU damage. A surprising result of some studies of excitotoxicity in cortical cultures was the inability of KA to kill neurons unless present in culture medium for hours (Choi et al., 1988). This observation markedly contrasted with those of others who found KA very toxic in their slice and culture preparations (Hajos et al., 1986; Frandsen and Schousboe, 1987; Rothman et al., 1987) and has not been explained. Finally, the role of ionized cytoplasmic calcium in producing cell death has not been resolved. Some experiments have suggested that the concentration of calcium predicts neuronal death after GLU exposure (Stodieck and Miller, 1987) while others have failed to confirm that this phenomenon generalizes to all cells (Lemasters et al., 1987).

The experiments described below were designed to resolve some of these questions. They have employed a recently synthesized, highly potent competitive antagonist of $\mathrm{KA}$ and QUIS receptors, 6-cyano-7-nitroquinoxaline-2,3-dione (CNQX; Honore et al., 1988), to investigate the role of non-NMDA receptors in producing GLU toxicity. These experiments also used the dye Fura-2 (Grynkiewicz et al., 1985) to obtain intracellular calcium concentrations in cells exposed to paradigms testing possible mechanisms of excitotoxicity. 


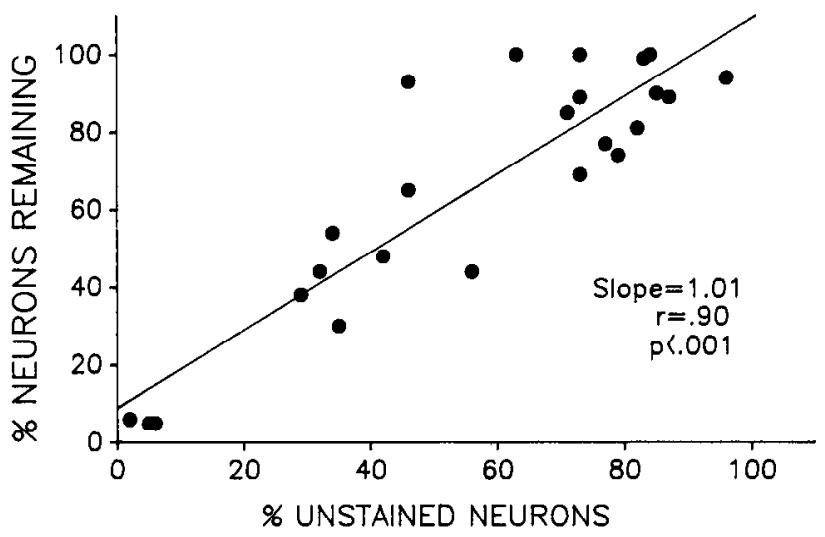

Figure 1. Percentage of unstained neurons provides a valid measure of neuronal survival. Fields of neurons were photographed under phasecontrast optics prior to incubation with control MEM or MEM + GLU \pm various excitatory amino acid antagonists and then relocated and photographed again after $18 \mathrm{hr}$. An additional bright-field photograph of the same field was taken after trypan blue staining. The percentage of unstained neurons ( $a b s c i s s a$ ) correlates well with the fraction of neurons still present after the experimental manipulation. The latter figure was obtained by counting neurons in the fields before, and $18 \mathrm{hr}$ after, GLU exposure.

\section{Materials and Methods}

Tissue culture. Dispersed hippocampal cultures were prepared using the methods of Huettner and Baughman (1986) with very minor modifications. Details of our culture protocol have already been published (Yamada et al., 1989). Cultures used for the experiments described below were between 14 and $18 \mathrm{~d}$ in vitro. The calcium determinations described below were done with identical cultures grown on glass coverslips glued into $35-\mathrm{mm}$ culture dishes from which the bottoms had been removed.

Electrophysiology. For intracellular recording, growth medium was replaced with a balanced salt solution containing (in $\mathrm{mM}$ ): $140 \mathrm{NaCl} ; 3$ $\mathrm{KCl} ; 4 \mathrm{CaCl}_{2} ; 4 \mathrm{MgCl}_{2} ; 10$ HEPES; and 5.5 glucose; $\mathrm{pH}$ was 7.3 . Tetrodotoxin $(1 \mu \mathrm{g} / \mathrm{ml})$ was added to eliminate regenerative sodium conductances. In experiments examining NMDA responses, $\mathrm{MgCl}_{2}$ was removed from the bath and $10 \mu \mathrm{M}$ glycine added (Johnson and Ascher, 1987). The cultures were placed on the stage of an inverted microscope and studied at room temperature. Cells were voltage clamped with lowresistance $(\sim 10 \mathrm{M} \Omega)$ pipettes using the whole-cell patch clamp technique (Hamill et al., 1981). Our intracellular solution contained (in mM): 130 CsAC; $10 \mathrm{CsCl} ; 10$ HEPES; 1.1 EGTA; and $2 \mathrm{Mg}$-ATP. Current signals were filtered at $0.2 \mathrm{kHz}$, digitized at $0.36 \mathrm{kHz}$, and stored on disk for later analysis.

We examined the effects of putative excitatory amino acid antagonists by comparing inward currents produced by brief application of an agonist with current seen after equivalent application of agonist in the presence of antagonist. Drugs were pressure applied $\left(2-7 \times 10^{4} \mathrm{pa}\right)$ from micropipettes with tip diameters approximately $1-2 \mu \mathrm{m}$, placed within $50 \mu \mathrm{m}$ of a voltage-clamped neuron (Choi and Fischbach, 1981). The drugs were always dissolved in a buffer identical to that bathing the cells. In most experiments, agonist (usually GLU) responses using the same pipette were compared before and immediately after a 4-10-sec local application of antagonist from a second pipette. At other times the current produced by agonist delivered from 1 pipette was compared with current produced by the simultaneous application of agonist and antagonist delivered from a second pipette. These methods have previously provided us with reliable, quantitative results (Yamada et al., 1989).

Toxicity. Growth medium was replaced with MEM without phenol (Earle's salts) modified to contain (in mM): $1 \mathrm{NaHCO}_{3}, 0.1$ glutamine, 0.01 glycine, 33 glucose, 35 sucrose, and 10 HEPES; pH was 7.3. The sucrose was added to increase the osmolarity to 350 mOSM to resemble our bicarbonate-buffered standard growth medium. In experiments examining potential protective effects of excitatory amino acid antagonists, the drugs were added to the dishes from stock solutions and allowed to equilibrate for $2 \mathrm{~min}$. At that time different excitatory amino acids were added and the cultures returned to a $37^{\circ} \mathrm{C}$ incubator for either $20 \mathrm{~min}$ or $2 \mathrm{hr}$. Cultures were then washed 3 times with Earle's balanced salt solution supplemented with $33 \mathrm{~mm}$ glucose and returned to a $37^{\circ} \mathrm{C}$ incubator containing 5\% carbon dioxide. They remained in the EBSS for $18 \mathrm{hr}$, when cell survival was assessed by dye exclusion. For this period the cells survived as well in EBSS as in MEM or in full media.

Trypan blue ( $4 \%$ final concentration) was added to cultures for $5 \mathrm{~min}$ and then removed by 2 washes with EBSS. Two $1-\mathrm{mm}^{2}$ areas of each dish, which had been scored prior to any experimental manipulation, were then counted under bright-field optics to give the number of dyestained neurons and total number of neurons in each field. Each experiment was replicated with at least 6 dishes (12 fields). We were conccrncd that this method might underestimate cell death if dead neurons disintegrated without leaving a dye-stained remnant. Therefore, in 24 dishes fields of neurons were photographed before an experiment and then relocated and photographed after $18 \mathrm{hr}$. They were then stained with trypan blue. The fraction of clearly identifiable, phase bright neurons that was initially present and remained at $18 \mathrm{~h}$ was compared with the fraction of unstained neurons determined with dye. Although there was some variability between the two measures, correlation was quite good (Fig. 1).

In 1 set of experiments we examined the potential toxicity of elevated extracellular potassium. The solution used in these experiments was identical to standard MEM except that $122 \mathrm{~mm} \mathrm{KCl}$ and $1 \mathrm{mM} \mathrm{KH}_{2} \mathrm{PO}_{4}$ were substituted equimolar for $\mathrm{NaCl}$ and $\mathrm{NaH}_{2} \mathrm{PO}_{4}$, respectively. The same additions made to our MEM listed above were also used in this solution.

Calcium measurements. Hippocampal neurons were loaded with 1.5 $\mu \mathrm{M}$ Fura-2/AM (Molecular Probes, Eugene, OR) added to the medium for $1-2 \mathrm{hr}$ at $37^{\circ} \mathrm{C}$. This medium was then removed with at least 3 washes of MEM without phenol, exactly like the medium used for the toxicity experiments. This solution was then left in the dishes for 15$30 \mathrm{~min}$ before any measurements were undertaken. Drugs and amino acids were added to the cultures from $100 \times$ stock solutions during calcium measurements. Small groups of cells (rarely individual cells) were examined on a Zeiss IM-35 microscope equipped for epifluorescence, using a Zeiss Plan 40 (NA $=0.65$, air) objective (Fig. $2, A, B$ ). During experiments, cells were maintained at $36-37^{\circ} \mathrm{C}$ on a thermostatically controlled heating stage. During excitation, 2 bandpass interference filters were alternated manually: one $340 \mathrm{~nm}$ (Ditric Optics, Hudson, MA) in series with an Inconel-coated neutral-density filter (density $=0.6$ ) (Harvard Apparatus, South Natick, MA), and the other, $380 \mathrm{~nm}$ (Corion Corp., Holliston, MA) in series with 2 Schott 0.6 neutral-density filters to minimize bleaching. Emitted light was collected at 480-530 nm (Schott colored glass, GG495) and focused on an intensified silicon intensifier target-tube camera (DAGE, MTI, model 66). The output was digitized by a Grinnell GMR 274 video frame buffer then transferred to a VAX $11 / 780$ computer. Ratio images were produced by dividing $340-\mathrm{nm}$ images by $380-\mathrm{nm}$ images, after the substration of background (neuron-free region) and autofluorescence (Fig. $2, C, D$ ). An intensity threshold was also set before the ratio was made in order to exclude noncellular regions from the determination of the ratio image. Images were stored on compact tapes (Digital TK50, Digital Equipment Corp.; 90-megabyte capacity). Fluorescence intensity was analyzed in multiple regions throughout a neuron, with each regional intensity value representing an average from 121 pixels of an $11 \times 11$ matrix $\left(4.2 \mu \mathrm{m}^{2}\right)$. Ratios from 2 to 5 regions were averaged to obtain a single ratio for each neuron.

We used the formula $\mathrm{Ca}=K_{\mathrm{D}}\left[\left(R-R_{\min }\right) /\left(R_{\max }-R\right)\right]\left(F_{\mathrm{O}} / F_{\mathrm{S}}\right)$ (Grynkiewicz et al., 1985) to convert our ratio images to free intracellular calcium concentrations $\left(\mathrm{Ca}_{\mathrm{i}}{ }^{2+}\right)$. The values for $R_{\min }, R_{\max }$, and $F_{\mathrm{O}} / F_{\mathrm{s}}$ were obtained by measuring the fluorescence of $20 \mu \mathrm{M}$ Fura-2 (potassium salt. Molecular Probes) in a solution of (in mM): $150 \mathrm{KCl}, 10 \mathrm{HEPES}$, $2 \mathrm{MgSO}_{4}, 10 \mathrm{EGTA}$, and $\mathrm{NaOH}$ to $\mathrm{pH} 7.1 . R_{\min }$ was the $340 / 380$ ratio without added calcium; $R_{\text {max }}$ was the ratio when saturating calcium, 15 mM, was added; and $F_{\mathrm{O}} / F_{\mathrm{s}}$ was the $380-\mathrm{nm}$ intensity in saturating calcium. For our microscope and camera, $R_{\min }=0.2, R_{\max }=4.6$; and $F_{\mathrm{O}} / F_{\mathrm{S}}=3.41$. We used the previously published $K_{\mathrm{D}}$ for Fura-2, $224 \mathrm{nM}$ (Grynkiewicz et al., 1985).

Amino acid antagonists. Dr. Tage Honoré of Ferrosan provided our initial sample of CNQX. Subsequent batches of CNQX were purchased from Tocris Neuramin. The methyl-10,11-dihydro-5-H-dibenzocyclohepten-5, 10-imine (MK-801) was a gift of Merck Sharp, and Dohme. We obtained D-2-amino-5-phosphonovalerate (APV) from either Cam- 

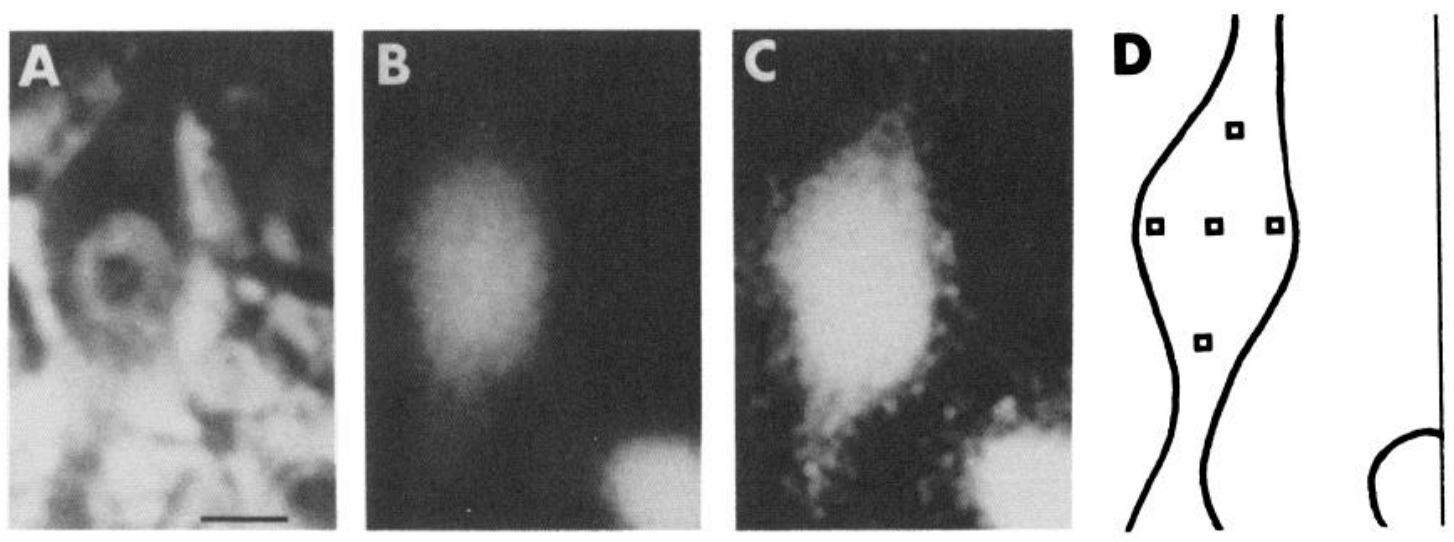

Figure 2. Sample Fura-2 ratio images. $A$, Phase image of 2 hippocampal neurons that were loaded with Fura-2. $B$, Epifluorescence image ( 380 $\mathrm{nm}$ ) of the same 2 neurons, showing the much greater fluorescence within the neurons than surrounding glia. $C$, Ratio image of same 2 cells created by image processor using 340- and 380-nm images. $D, 340 / 380$ ratios for individual cells were obtained by averaging $11 \times 11$ matrices containing 121 pixels from several portions of the soma and/or proximal dendrites. These values were then averaged to provide an overall ratio for individual neurons within a field. The line drawing shows the 5 regions used in the larger neuron in this figure. Scale bar, $A-D, 7 \mu \mathrm{m}$.

bridge Research Biochemicals or Tocris Neuramin. The excitatory amino acids were purchased from Sigma.

\section{Results}

\section{Electrophysiology}

Although other investigators have shown that CNQX is a potent antagonist of non-NMDA excitatory amino acid receptors (Honoré et al., 1988; Neuman et al., 1988; Yamada et al., 1989), we wanted to establish that CNQX could significantly inhibit inward currents produced by the application of toxic concentrations of GLU. For short-term exposures this GLU concentration is $500 \mu \mathrm{M}$ to $1 \mathrm{~mm}$ (Choi et al., 1987; Rothman et al., 1987). This required a CNQX concentration high enough to antagonize $500 \mu \mathrm{M}$ GLU acting at non-NMDA receptors. However, at high concentrations, CNQX is a noncompetitive inhibitor of the NMDA receptor at the glycine site (Yamada et al., 1989). Therefore, an excessive CNQX concentration would block all excitatory amino acid responses. We found that $200 \mu \mathrm{M}$ CNQX very strongly blocked GLU currents without totally eliminating responses to NMDA itself.

Brief $(100 \mathrm{msec})$ applications of $500 \mu \mathrm{M}$ GLU to voltageclamped neurons produced an inward current that was almost completely eliminated by $200 \mu \mathrm{M}$ CNQX (Fig. $3 A$ ). In 5 consecutive neurons the reduction in GLU current was $85.6 \pm 3.3 \%$ (SEM).

In our neurons, inward currents produced by $500 \mu \mathrm{M}$ NMDA were diminished by $75.7 \pm 3.3 \%(n=6$; Fig. $3 B)$. However, the absolute magnitude of the inward current produced by 500 $\mu \mathrm{M}$ GLU acting at the NMDA receptor will be larger than that elicited by $500 \mu \mathrm{M}$ NMDA itself, owing to the higher affinity of the former for the NMDA receptor (Olverman et al., 1984). APV, $1 \mathrm{mM}$, diminished GLU currents by $21.3 \pm 4.2 \%(n=7$; not shown). This probably reflects the small NMDA component of GLU currents produced by high GLU concentrations. MK$801,20 \mu \mathrm{M}$, had little effect on GLU currents (Fig. 3C), as expected from other reports showing virtually no effect of MK801 on KA/QUIS responses (Huettner and Bean, 1988). Repeat applications of MK-801 might have made the NMDA component of the GLU currents more evident.

\section{Neurotoxicity}

A 20 -min exposure to $500 \mu \mathrm{M}$ GLU killed $75 \%$ of our cultured hippocampal neurons (Figs. $4 B, 5 A$ ). In the presence of $200 \mu \mathrm{M}$ CNQX, added prior to the GLU, the toxicity of GLU was only minimally attenuated, to $64 \%$ (Figs. $4 C, 5 A$ ). Thus, concentrations of CNQX capable of attenuating $85 \%$ of the inward current
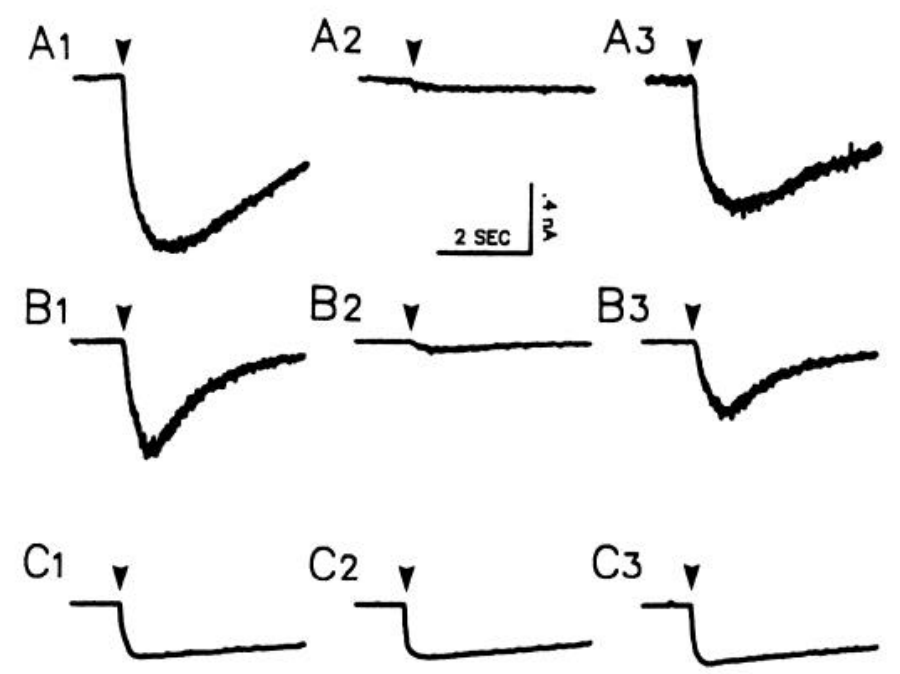

Figure 3. CNQX blocks GLU- and NMDA-induced inward currents in voltage-clamped hippocampal neurons. $A$, The current produced by a 100-msec application of $500 \mu \mathrm{M}$ GLU (arrowhead, A1) was almost completely eliminated when $200 \mu \mathrm{M}$ CNQX was locally perfused for 5 $\mathrm{sec}$ before the GLU application (A2). After $30 \mathrm{sec}$ the GLU response almost completely recovered $(A 3)$. The neuron was clamped at -60 $\mathrm{mV}$ and the current filtered at $0.2 \mathrm{kHz}$. B, Current elicited by a $500-$ msec exposure to $500 \mu \mathrm{M}$ NMDA $(B I)$ was also reduced by prior application of $200 \mu \mathrm{M}$ CNQX (B2). This was completely reversible (B3). NMDA experiments were done in the absence of added magnesium and in the presence of $10 \mu \mathrm{M}$ glycine; cells were held at $-50 \mathrm{mV}$. $C$, Inward current after a $200-\mathrm{msec}$ GLU pulse $(C 1)$ was not reduced when GLU was simultaneously applied with $20 \mu \mathrm{M}$ MK-801 (C2). Reexposure to GLU alone $(C 3)$ shows no residual effects of MK-801. Cell clamped at $-60 \mathrm{mV}$. 

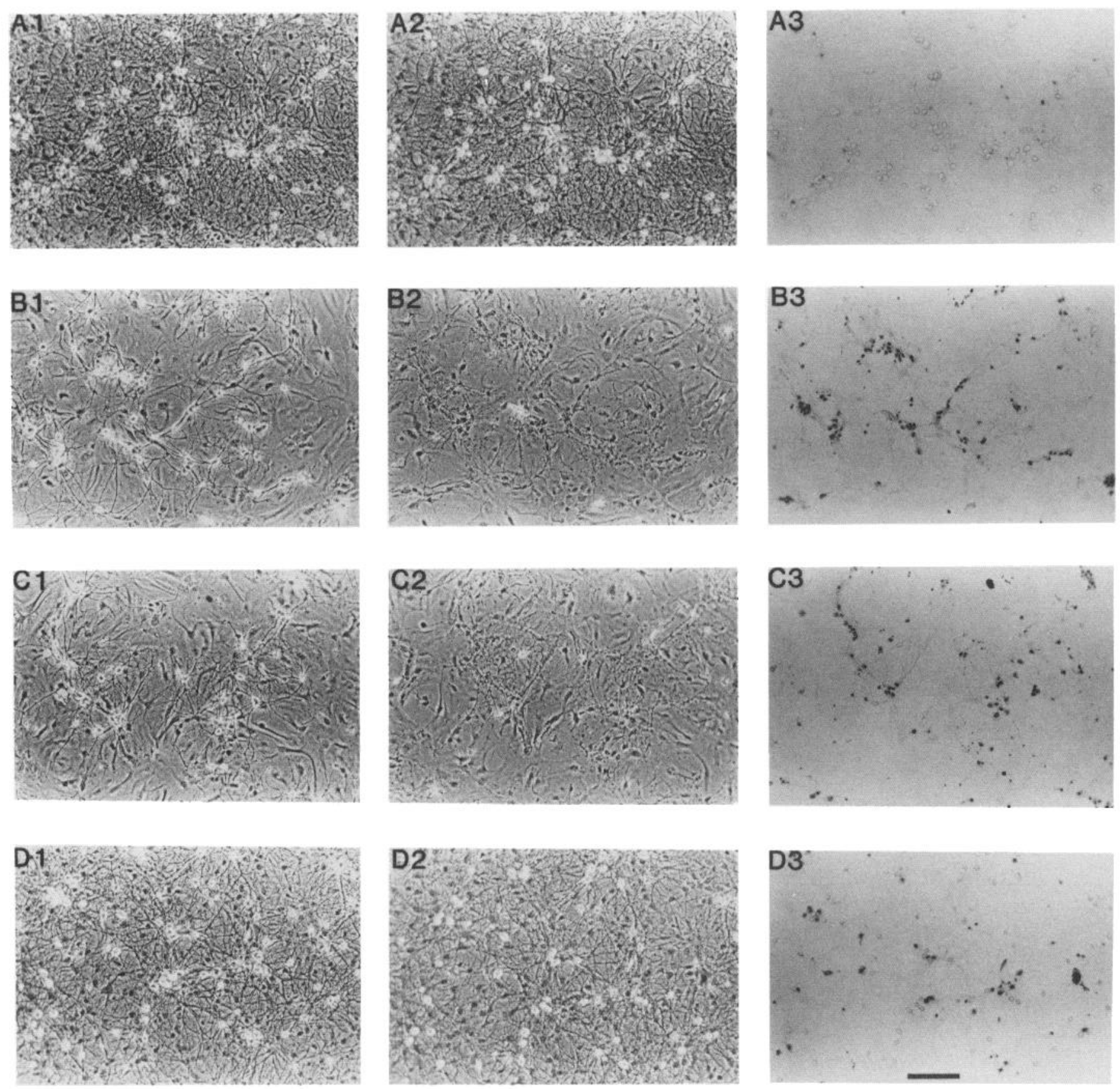

Figure 4. CNQX does not block GLU neurotoxicity. $A$, Three photographs showing neurons in a single microscope field before $(A 1)$ and $18 \mathrm{hr}$ after a 20-min medium change to MEM without GLU $(A 2, A 3) . A 1$ and $A 2$ are phase-contrast photographs showing the cell bodies of typical phasebright neurons. $A 3$ is a bright-field photograph showing that very few neurons have taken up the trypan blue vital stain. $B$, Field in a sister culture shown before $(B 1)$ and $18 \mathrm{hr}$ after a 20 -min incubation with $500 \mu \mathrm{M}$ GLU $(B 2, B 3)$. Under bright field most neurons are stained with trypan blue (B3). $C$, Sequence identical to $A$ and $B$ showing field from another culture pretreated with CNQX prior to GLU addition. The trypan blue staining (C3) shows that most neurons are no longer viable. $D$, Pretreatment with APV leads to some decrease in GLU toxicity, but a number of neurons are still trypan blue stained after the GLU + APV exposure (D3). Scale, $A-D, 200 \mu \mathrm{m}$.

produced by GLU had an insignificant influence on GLU toxicity. CNQX was not toxic on its own. High concentrations of APV, $1 \mathrm{~mm}$, were more protective than CNQX, but still allowed over $50 \%$ of the neurons to die after GLU exposure (Figs. $4 D$, $5 A$ ). MK-801, which did not alter GLU currents at $20 \mu \mathrm{M}$, preserved almost all the neurons after a 20-min GLU exposure (Figs. 5A, 6A). In fact, cell death after the combination of GLU + MK-801 (14\%) was about half of control cell loss (27\%). This likely represents a small amount of cell death in controls from stimulation of endogenous GLU release, which has been described in other culture preparations (Hahn et al., 1988).

The inability of CNQX to protect these cultured neurons from GLU toxicity indicated that KA/QUIS receptors were relatively unimportant for this type of toxicity, as has been suggested previously (Choi et al., 1988). However, as other investigators and ourselves have found substantial KA toxicity in dispersed 
neuronal cultures (Frandsen and Schoushoe, 1987; Rothman et al., 1987), we also examined the effects of high concentrations of $\mathrm{KA}$ in our preparation. A 20 -min incubation in $1 \mathrm{~mm} \mathrm{KA}$ killed $60 \%$ of our neurons, indicating that, at least at high concentrations, KA was very toxic (Figs. $5 B, 6 B$ ). Unexpectedly, we found that this toxicity was markedly attenuated by adding $20 \mu \mathrm{M}$ MK-801 prior to KA exposure (Figs. $5 B, 6 C$ ). Release of endogenous GLU does not play a major role in this $\mathrm{KA}$ toxicity, as incubating the cells in $123 \mathrm{~mm} \mathrm{KCl}$ for $20 \mathrm{~min}$ did not produce much cell death (Fig. $5 \mathrm{C}$ ). The cell death seen with the potassium exposure was also not reduced by MK-801 (Fig. $5 C$ ). We suspect that much of the KA toxicity results from nonspecific activation of NMDA channels by KA (Jahr and Stevens, 1987; Cull-Candy and Usowicz, 1987).

We were concerned that the MK-801 protection from GLU toxicity might be only short lived, and that the dramatic swelling seen in our neurons exposed to GLU, even in the presence of MK-801, would eventually prove deleterious if maintained for more than $20 \mathrm{~min}$. However, even when the exposure was prolonged to $2 \mathrm{hr}, \mathrm{MK}-801$ was still protective. Glutamate alone now killed $89.9 \pm 2.1 \%$ (SEM; $n=14$ fields) of the neurons, but when MK-801 was present, mortality dropped to $29 \pm 3 \%$. Control cell death in this case was $26.3 \pm 2.6 \%$.

\section{Intracellular calcium and neurotoxicity correlation}

Hippocampal cultures grown on glass coverslips were preloaded with Fura-2/AM and placed on the heated stage of an inverted microscope, but otherwise treated identically to cultures used in the neurotoxicity experiments. Neurons, typically in clusters of 4-6 cells, were imaged with $340 \mathrm{~nm}$ and $380 \mathrm{~nm}$ fluorescence to provide an intensity ratio. Prior to the addition of any amino acid agonist or antagonist, we obtained control ratios on up to 10 neurons in a culture. The imaging was repeated after 15-20 min, the time interval in the toxicity experiments, on the same or occasionally nearby neurons. In preliminary experiments, we saw no change in the $340 / 380$ ratio in clusters of neurons monitored over $45 \mathrm{~min}$ without any drug additions (Fig. $8 A$ ). The $340 / 380$ ratio in these neurons prior to any drug addition was 0.45 ; addition of $500 \mu \mathrm{M}$ GLU elevated this ratio to 1.64. In the presence of CNQX, APV, or MK-801, GLU did not significantly increase the ratio over control (Fig. $7 A$ ). KA also produced a substantial elevation of the $340 / 380$ ratio which was partially reduced by pretreatment with MK-801. Elevated extracellular potassium also increased the ratio (Fig. $7 A$ ).

While there was a tendency for paradigms associated with a large degree of neuronal death to produce significant elevations of the $340 / 380$ image after $20 \mathrm{~min}$, the correlation was weak (Fig. $7 B$ ). Plotting the ratio against the percent neuronal loss (taken from Fig. 5) produced a correlation coefficient of 0.41 , which was not significantly greater than zero.

As the 20-min 340/380 ratio correlated poorly with cell survival, we examined the ratio over shorter time periods in some cells monitored serially for up to $45 \mathrm{~min}$. Not unexpectedly, ratios in neurons treated with GLU alone remained elevated during the entire cxposurc (Fig. $8 A$ ). Neurons trcated with APV prior to GLU showed a transient peak at $5 \mathrm{~min}$, which decayed back to near control values at $15 \mathrm{~min}$. CNQX pretreatment even prevented this early peak despite being ineffective in protecting neurons from GLU toxicity.

We also investigated the effects of the amino acid antagonists on $\mathrm{Ca}_{\mathrm{i}}{ }^{2+}$ to make sure that the ratios were not influenced by drug treatment alone. Neurons were imaged prior to drug ad-

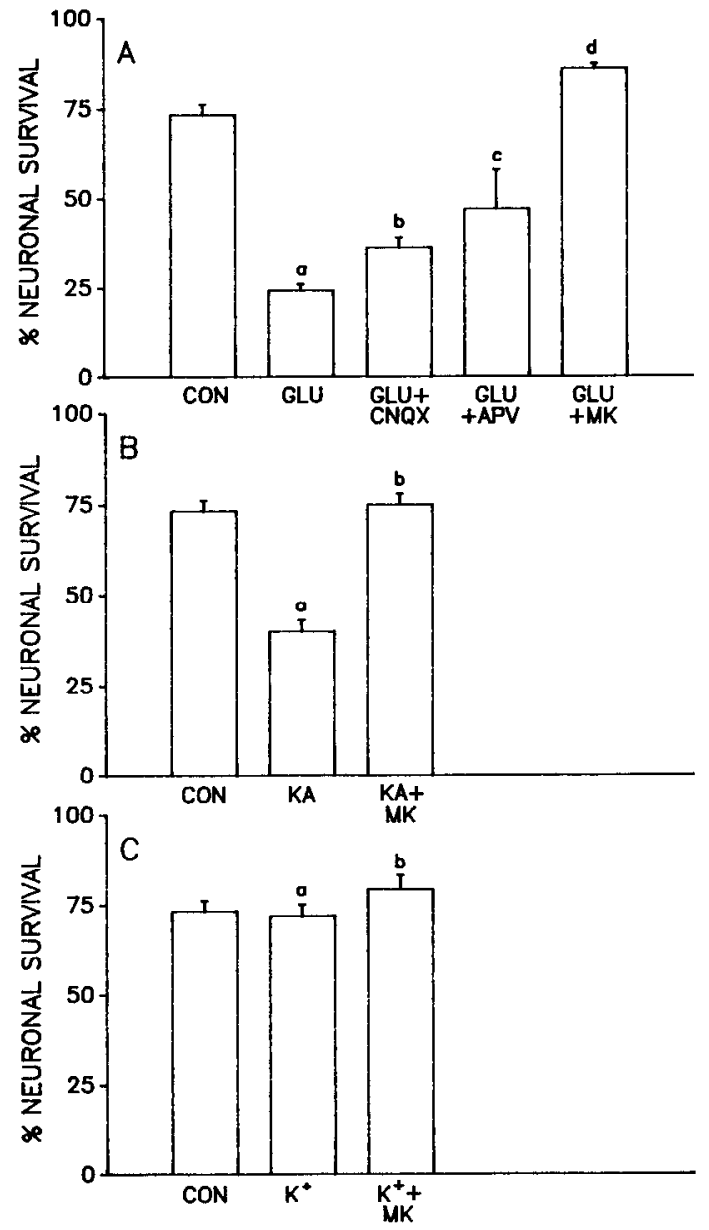

Figure 5. Quantitative neurotoxicity of the different experimental paradigms. $A$, Percent neuronal survival seen $18 \mathrm{hr}$ after a 20 -min GLU exposure in the absence or presence of various antagonists. $a$, GLU survival worse than control (CON), APV, and MK-801 $(M K)(p<0.01$ by Student-Newman-Keuls test) but not different from CNQX $(p>$ $0.05) ; b$, CNQX survival different from CON and MK $(p<0.01)$ but not GLU or APV ( $p>0.05) ; c$, APV different from GLU, CON, and MK $(p<0.01)$; $d$, MK different from all groups $(p<0.01)$ except CON. $B$, Survival after KA exposure. $a, \mathrm{KA}$ is worse than either control or KA + MK-801 ( $p<0.01$ by Student-Newman-Keuls test); $b$, not different from CON. $C$, Effect of 20 -min incubation in $123 \mathrm{~mm}$ potassium $\left(\mathrm{K}^{+}\right)$on neuronal survival. $a, b, \mathrm{~K}^{+}$, with or without $\mathrm{MK}-801$, is no different than CON $(p>0.05)$. Error bars represent SEM. Values are averages of 12 fields in 6 separate cultures except for both sets of KA data ( 18 fields in 9 cultures) and the APV data ( 11 fields in 11 cultures).

dition and after 15 min of incubation in drug but before exposure to GLU or KA. Both CNQX and APV decreased intracellular calcium (Fig. $8 B$ ). Interestingly, MK-801 significantly elevated the $340 / 380$ ratio.

In view of evidence linking hypoxic neuronal injury to increases in extracellular GLU (Rothman and Olney, 1986), we also did 1 set of experiments to determine whether hypoxia in our cultures increased $\mathrm{Ca}_{i}{ }^{2+}$ and whether this increase could be prevented by GLU antagonists. Hypoxia was produced chemically by adding $4 \mathrm{~mm} \mathrm{NaCN}$ to cultures treated identically to those described above. $\mathrm{NaCN}$ treatment alone elevated the $340 /$ 380 ratio to levels comparable to those seen with $500 \mu \mathrm{M}$ GLU incubation (Fig. 9). Both APV and CNQX pretreatment substantially attenuated this increase in intracellular calcium. In 

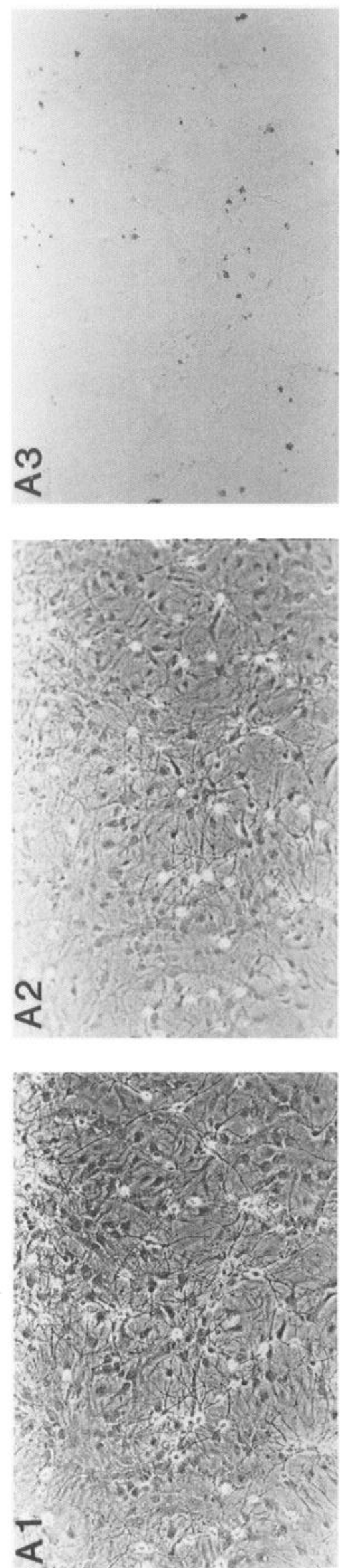
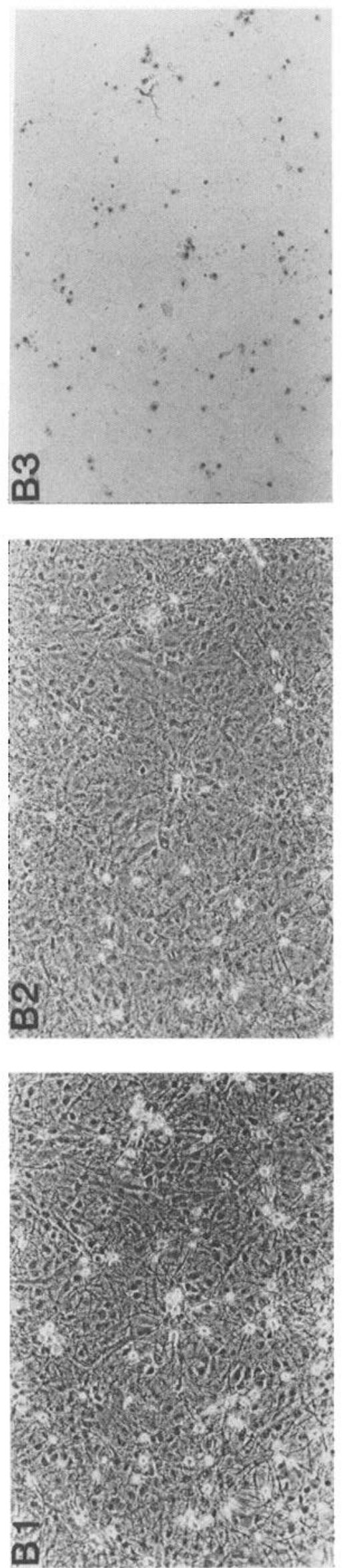
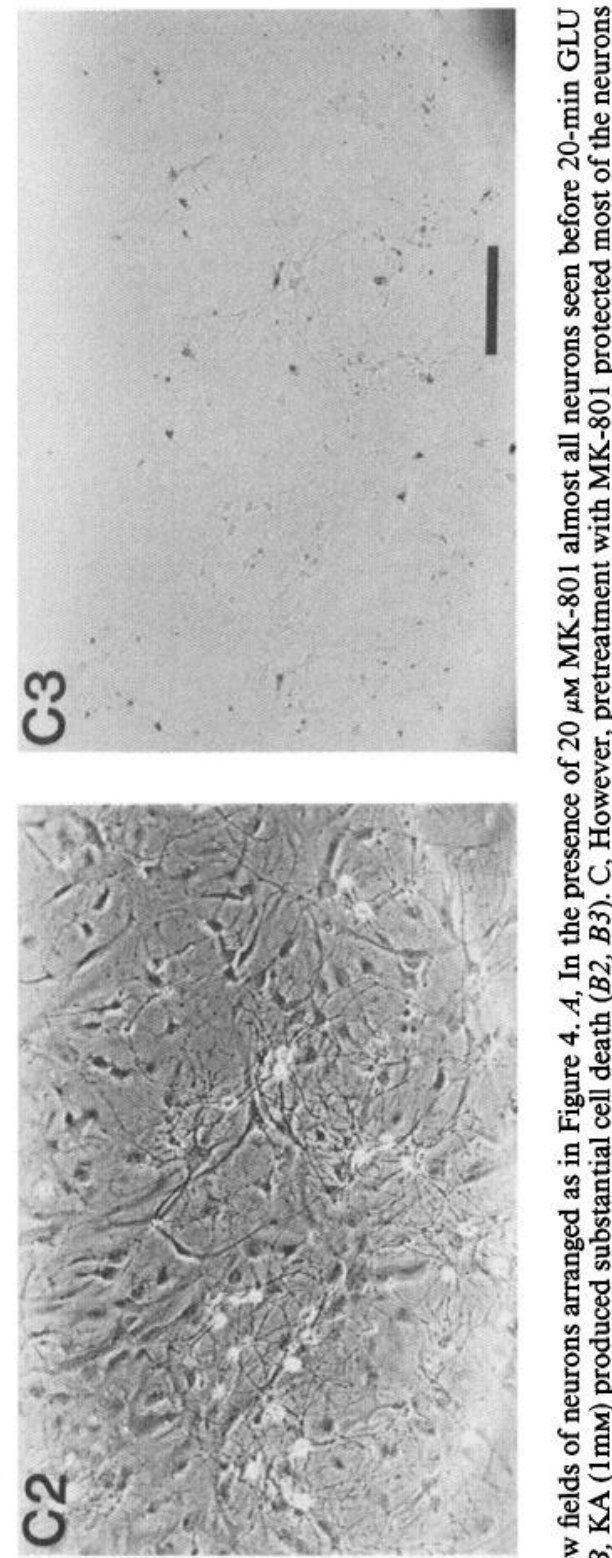

ㄴ.

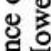

就定

范

迹

కกี

명

음

호융

.

s

J

影

5

룽

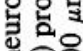

등 을

प

전

药

(c)

पेत्रु

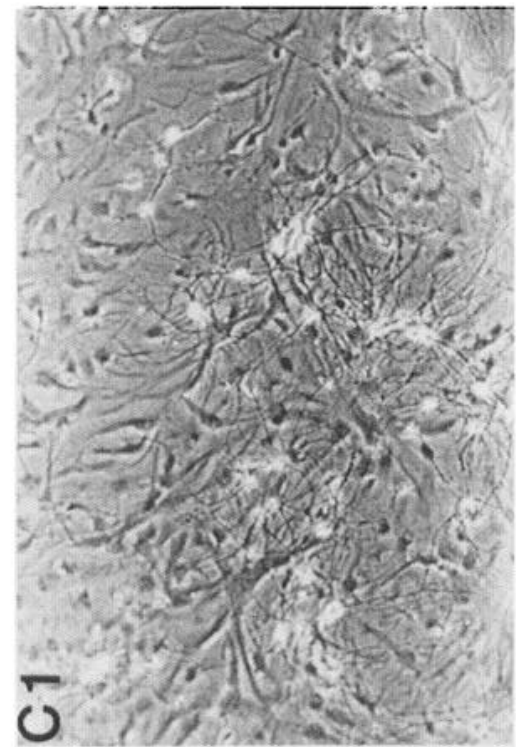

पै

उ둥

은

ส

बृ

这

o.

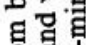

옹

s

웅형

-

这若

늠

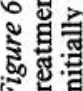



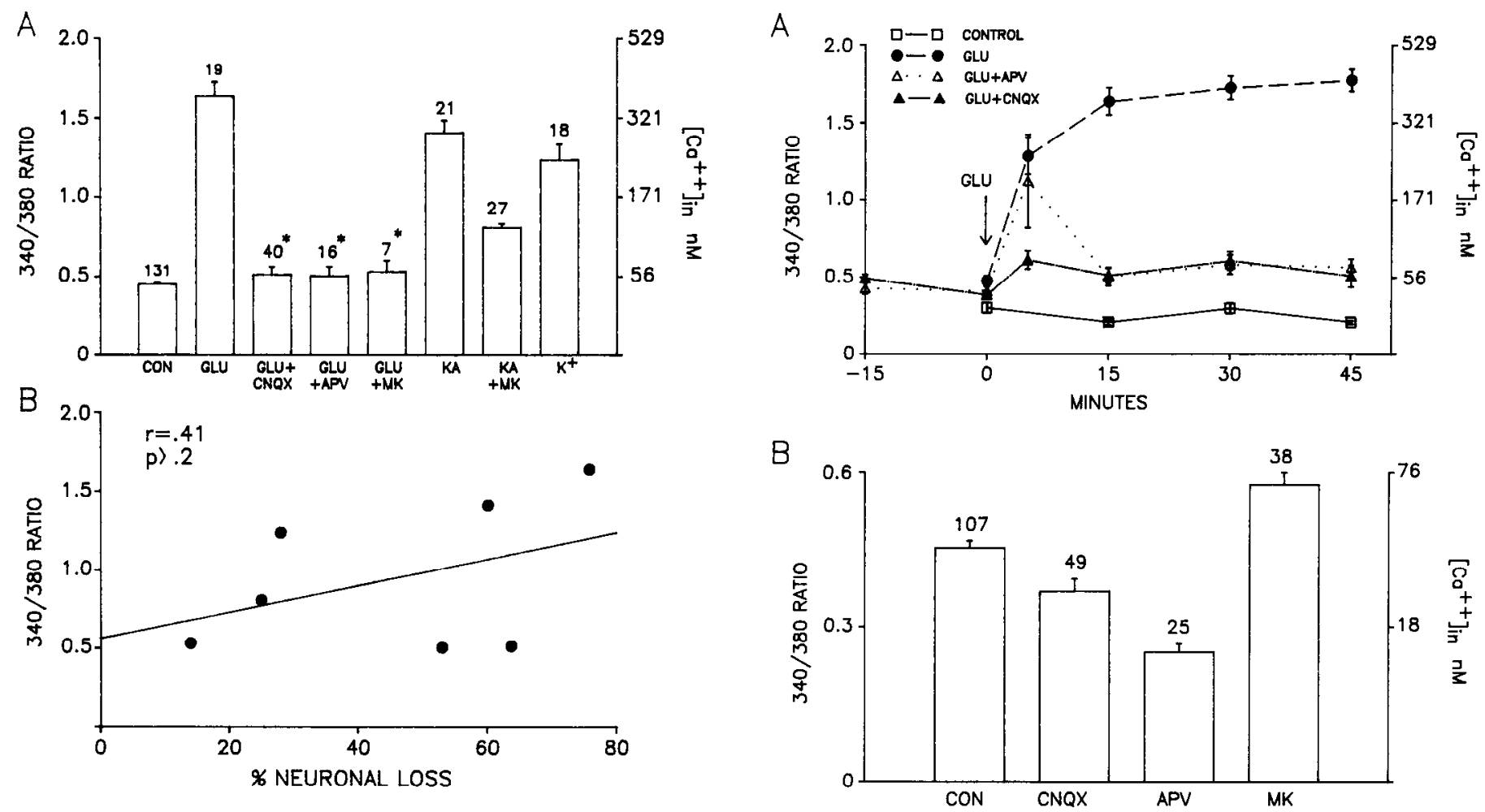

Figure 7. Intracellular calcium and neuronal death. $A, 340 / 380$ ratios calculated for individual neurons after 15-20-min exposure to various experimental solutions. $C O N$ represents the average initial ratio for 131 neurons prior to any drug additions. Numbers above the bars are sample size. Asterisks signify no difference from control $(p>0.05$ by StudentNewman-Keuls test). Error bars are SEM. B, The 340/380 ratios in $A$ plotted against the neuronal loss shown in Figure 5 . There are only 7 points because control is not included in the regression. While there is a correlation between calcium and neuronal loss, it is weak and not significantly greater than zero $(t$ test).

the presence of combined APV and CNQX, there was virtually no increase in the calcium measurements.

\section{Discussion}

The experiments described above clarify some issues related to the neurotoxicity of GLU and identify others that still require elucidation. In agreement with Choi and his colleagues (1988), GLU neurotoxicity in cultured central neurons depends largely on activation of the NMDA receptor. We were concerned that the non-NMDA antagonists used in their previous studies were too weak to adequately block KA and QUIS receptors and, therefore, did not show much of a KA/QUIS component to GLU toxicity. However, in the present study, CNQX was not able to significantly block GLU toxicity even though it eliminated almost $90 \%$ of the inward current produced by a neurotoxic GLU concentration. It is possible that even higher CNQX concentrations would increase survival, but these would also antagonize the NMDA component of GLU neurotoxicity. APV was not particularly successful in blocking GLU toxicity in our experiments, most likely because even $1 \mathrm{~mm}$ of the active D isomer was still an inadequate concentration to block $500 \mu \mathrm{M}$ GLU acting at NMDA receptors for 20 min (Olverman et al., 1984). MK-801 was more successful because its block of NMDA currents is noncompetitive (Wong et al., 1986; Huettner and Bean, 1988).

We were also concerned that the NMDA component of GLU

Figure 8. Effects of GLU and antagonists on intracellular calcium. $A$, Sequential intracellular calcium changes after exposure to GLU \pm antagonists. The " -15 to 0 " interval represents equilibration with APV or CNQX prior to GLU. Interestingly, the 340/380 ratio remains low for the entire 45-min exposure to GLU + CNQX and most of the exposure to GLU + APV despite the eventual loss of neurons. Control represents the $340 / 380$ ratio for neurons observed over 45 min but not exposed to GLU or antagonists. Bars are SEM. $B$, Effects of amino acid antagonists on intracellular calcium. Both CNQX and APV reduce the $340 / 380$ ratio $(p<0.01$ compared with control by Student-NewmanKeuls test), while MK-801 elevates it $(p<0.01)$. Numbers above bars are sample size.

toxicity might become relatively unimportant if the GLU exposure was prolonged. However, even lengthening the GLU incubation to $2 \mathrm{hr}$ did not diminish the ability of MK-801 to protect these neurons. Under these conditions the neurons noticeably swelled, but this was reversible with removal of the GLU. Thus, even in monolayer culture, neurons can undergo prolonged swelling and still survive, as has been shown for shorter periods (Choi et al., 1988). We did not previously appreciate the reversibility of neuronal swelling and, therefore, overemphasized an osmotic component of excitotoxicity in an earlier paper (Rothman, 1985).

There are likely situations in which non-NMDA receptors do play an important role in GLU toxicity. A population of cortical neurons that stains for NADPH-diaphorase is very sensitive to KA and QUIS toxicity and might be more vulnerable to GLU acting at non-NMDA receptors (Koh and Choi, 1988). The developing rat cerebellum has subpopulations of neurons with low NMDA and high KA sensitivity (Garthwaite and Garthwaite, 1986). There are also neurons in the retina that are extremely susceptible to both NMDA- and non-NMDA-mediated excitotoxicity (Price et al., 1988). Recognizing all this, it is still significant that KA toxicity in our cultured hippocampal neurons was reduced by $\mathrm{MK}-801$. This implies that at least in some cascs KA toxicity is due to nonspecific activation of NMDA 


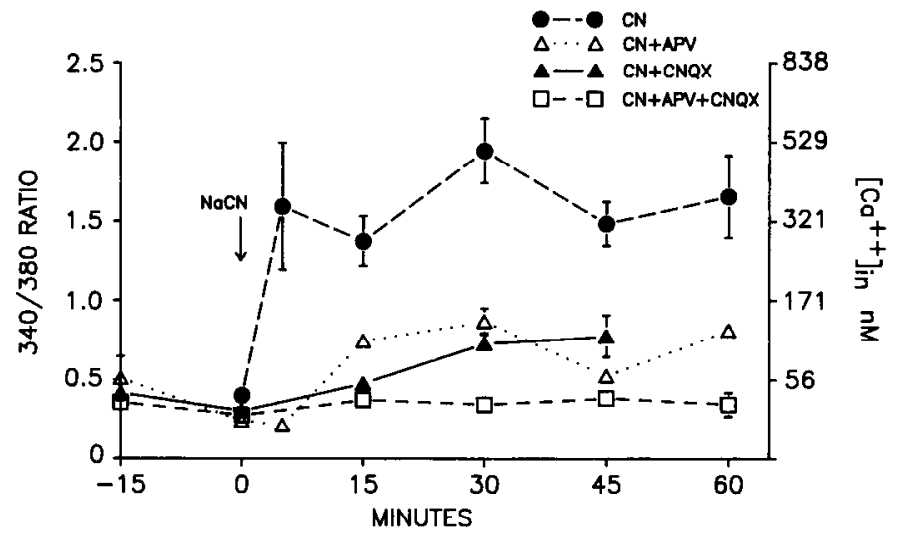

Figure 9. Amino acid antagonists block intracellular calcium elevations produced by "chemical hypoxia." Both $\Lambda P V$ and CNQX individually suppress the $340 / 380$ ratio increase produced by $4 \mathrm{~mm} \mathrm{NaCN}$. The 2 combined entirely prevent any rise. In the " -15 to 0 " interval the decrease due to drug addition is apparent. Error bars are SEM.

receptors, as supported by single channel records (Cull-Candy and Usowicz, 1987; Jahr and Stevens, 1987). Alternative hypothcses, KA inhibition of cndogenous GLU reuptakc or a unique enhancement of GLU release, still cannot be completely eliminated (Pocock et al., 1988). The blockade of KA toxicity by MK-801, which has little effect on KA-induced depolarizations, and the failure of depolarizing concentrations of potassium to kill our neurons again point out the small role of voltage-gated calcium channels in this type of neurotoxicity. However, Mattson and his colleagues (1989) have come to a different conclusion about the pathophysiology of GLU neurotoxicity in hippocampal neurons less than 1 week in vitro. GLU likely kills these neurons via depolarization mediated by $\mathrm{KA} / \mathrm{QUIS}$ receptors and subsequent calcium entry. NMDA receptors do not play much of a role in cell death in these younger cultures. Thus, neuronal maturity can play a major role in determining the mechanism(s) by which GLU kills cells.

The results of the calcium determinations were not exactly as anticipated. We agree with others (Connor et al., 1987; Mayer et al., 1987; Murphy et al., 1987; Stodieck and Miller, 1987; Baimbridge and Kao, 1988) that GLU is clearly capable of elevating $\mathrm{Ca}_{i}{ }^{2+}$. However, we failed to fird the excellent correlation between $\mathrm{Ca}_{i}{ }^{2+}$ increase and cell death reported by different laboratories (Stodieck and Miller, 1987; Ogura et al., 1988; Mattson et al., 1989). Some treatments (CNQX, APV) that blocked $\mathrm{Ca}_{\mathrm{i}}{ }^{2+}$ elevations failed to protect from GLU. Other paradigms that led to $\mathrm{Ca}_{\mathrm{i}}{ }^{2+}$ elevations (KA + MK-801, $123 \mathrm{mM}$ $\mathrm{K}^{+}$) were relatively nontoxic. Sampling bias is an unlikely explanation for these $\mathrm{Ca}_{i}{ }^{2+}$ results. We almost always selected neurons in clusters for imaging and these are the cells most likely to die after toxic GLU exposure. There was insignificant variability among the cells in baseline $\mathrm{Ca}_{\mathrm{i}}{ }^{2+}$ values.

$A$ review of other recent reports suggests that $\mathrm{Ca}_{i}{ }^{2+}$ measurements may not necessarily predict neuronal death. Murphy and Miller $(1987,1988)$ have described $\mathrm{Ca}_{\mathrm{i}}{ }^{2+}$ elevations produced in cultured striatal neurons by $\mathrm{KA}$ that are as large as those produced by NMDA. These KA-induced $\mathrm{Ca}_{\mathrm{i}}{ }^{2+}$ increases were seen in the presence of MK-801, as were the $\mathrm{Ca}_{i}{ }^{2+}$ changes in our hippocampal neurons. If $\mathrm{Ca}_{\mathrm{i}}{ }^{2+}$ elevations are the main determinant of neuronal death, KA should have been a potent toxin in vitro even in the presence of MK-801. An elegant study of hepatocytes dying from inhibition of metabolism also failed to show a rise in free $\mathrm{Ca}_{\mathrm{i}}{ }^{2+}$ until membrane rupture (Lemasters et al., 1987). Experiments attempting to correlate neuronal death and the uptake of radioactive calcium, ${ }^{45} \mathrm{Ca}^{2+}$, have thus far provided conflicting results. Two recent studies of hypoxia (Goldberg et al., 1989) and GLU toxicity (Kurth et al., 1989) in cultured neocortex demonstrated an excellent correlation bctween number of neurons dying and uptake of ${ }^{45} \mathrm{Ca}^{2+}$. However, others have found that drugs (phenytoin, verapamil, diltiazem) that block ${ }^{45} \mathrm{Ca}^{2+}$ sequestration in similar hypoxic cultures still fail to prevent neuronal death (Weber et al., 1988).

The most reasonable interpretation of these and previous results (Choi, 1987; Rothman et al., 1987) is that calcium is important for excitotoxic cell death. This has been postulated as a general mechanism of "toxic cell death" for over 10 years (Schanne et al., 1979) and emphasized as an important factor in the death of neurons damaged by a variety of insults (Simon et al., 1984; Kass and Lipton, 1986; Siesjö, 1988). However, this toxic calcium overload may not be accurately reflected by a sustained elevation of $\mathrm{Ca}_{\mathrm{i}}{ }^{2+}$. Calcium may quickly enter other cellular compartments or bind to structures without necessarily remaining in solution in the cytosol. This could be the mechanism by which calcium damages cells. The in vivo observation that NMDA agonists, including GLU, produce a much greater drop of extracellular calcium in cerebral cortex than KA or QUIS (Pumain et al., 1987) supports the hypothesis that there are very significant differences in calcium fluxes caused by different excitatory amino acids which may not be accurately monitored by $\mathrm{Ca}_{i}{ }^{2+}$ measurements.

Other interpretations of our data and those of Murphy and Miller $(1987,1988)$ are also possible. Both of us visualized Fura signals only in the neuronal cell body and most proximal dendrites. If $\mathrm{Ca}_{\mathrm{i}}{ }^{2+}$ rose to much higher levels in distal dendrites than within the cell body, neither of us would have detected it. It is possible that this explains our failure to see a significant rise in $\mathrm{Ca}_{\mathrm{i}}{ }^{2+}$ in neurons treated with GLU + CNQX or GLU + APV. In addition, in our experiments $\mathrm{Ca}_{i}{ }^{2+}$ was monitored on an extremely slow time scale. We could easily have missed earlier transient $\mathrm{Ca}_{i}{ }^{2+}$ elevations, which may be important for cell damage. These questions require further investigation. We also did not monitor $\mathrm{Ca}_{\mathrm{i}}{ }^{2+}$ over many hours after GLU exposure. It is likely that $\mathrm{Ca}_{i}{ }^{2+}$ does rise immediately prior to cell death.

Two other findings require comment. First, APV and CNQX both produced small but statistically significant reductions in intracellular calcium. This may reflect antagonism of endogenous GLU always present in our medium although other investigations have seen unexplained increases in $\mathrm{Ca}_{\mathrm{i}}{ }^{2+}$ after exposure to competitive excitatory amino acid antagonists (Kudo and Ogura, 1986; Murphy et al., 1987). Even more interesting was the increase in $\mathrm{Ca}_{\mathrm{i}}{ }^{2+}$ seen after exposure to $20 \mu \mathrm{M}$ MK-801. While the mechanism for this is undetermined, it may be related to the cerebral metabolic changes seen in whole animals after systemic MK-801 administration (Nehls et al., 1988). This clearly requires further investigation.

Second, APV, CNQX, or the combination of both dramatically reduced the $\mathrm{Ca}_{\mathrm{i}}{ }^{2+}$ elevations seen in our neurons after $\mathrm{CN}$ exposure. This implies that a major component of the $\mathrm{Ca}_{\mathrm{i}}{ }^{2+}$ increase seen in neurons after hypoxia and even ischemia is caused by accumulation of endogenous GLU or ASP and will be reduced by antagonists of these excitatory amino acids. This observation further supports the link between excitatory amino 
acids and some types of hypoxic/ischemic brain injury first suggested 30 years ago (Van Harreveld, 1959).

\section{References}

Baimbridge, K. G., and J. Kao (1988) Calbindin D-28K protects again glutamate induced neurotoxicity in rat CAl pyramidal neuron cultures. Abstr. Soc. Neurosci. 14(2): 1264.

Choi, D. W. (1987) Ionic dependence of glutamate neurotoxicity in cortical cell culture. J. Neurosci. 7: 380-390.

Choi, D. W., and G. D. Fischbach (1981) GABA conductance of chick spinal cord and dorsal root ganglion neurons in cell culture. J. Neurophysiol. 45: 605-620.

Choi, D. W., M. A. Maulucci-Gedde, and A. R. Kriegstein (1987) Glutamate neurotoxicity in cortical cell culture. J. Neurosci. 7: 369379.

Choi, D. W., J. Y. Koh, and S. Peters (1988) Pharmacology of glutamate neurotoxicity in cortical cell culture: Attenuation by NMDA antagonists. J. Neurosci. 8: 185-196.

Collins, R. C., and J. W. Olney (1982) Focal cortical seizures cause distant thalamic lesions. Science 218: 177-179.

Connor, J. A., H. Tseng, and P. E. Hockberger (1987) Depolarization and transmitter-induced changes in intracellular $\mathrm{Ca}^{2+}$ of rat cerebellar granule cells in explant cultures. J. Neurosci. 7: 1384-1400.

Coyle, J. T. (1983) Neurotoxic action of kainic acid. J. Neurochem. 41: $1-11$.

Cull-Candy, S. G., and M. M. Usowicz (1987) Multiple-conductance channels activated by excitatory amino acids in cerebellar neurons. Nature 325: 525-528.

Finkbeiner, S., and C. F. Stevens (1988) Application of quantitative measurements for assessing glutamate neurotoxicity. Proc. Natl. Acad. Sci. USA 85: 4071-4074.

Frandsen, A., and A. Schousboe (1987) Time and concentration dependency of the toxicity of excitatory amino acids on cerebral neurons in primary culture. Neurochem. Int. 10: 583-591.

Garthwaite, G., and J. Garthwaite (1986) In vitro neurotoxicity of excitatory amino acid analogues during cerebellar development. Neuroscience $17: 755-767$

Garthwaite, G., F. Hajos, and J. Garthwaite (1986) Ionic requirements for neurotoxic effects of excitatory amino acid analogues in rat cerebellar slices. Neuroscience 18: 437-447.

Goldberg, M. P., R. G. Gifford, M. C. Kurth, and D. W. Choi (1989) Role of extracellular calcium and magnesium in ischemic neuronal injury in vitro. Neurology 39(Suppl. 1): 217.

Grynkiewicz, G., M. Poenie, and R. Y. Tsien (1985) A new generation of $\mathrm{Ca}^{2+}$ indicators with greatly improved fluorescence properties. J. Biol. Chem. 260: 3440-3450.

Hahn, J. S., E. Aizenman, and S. Lipton (1988) Central mammalian neurons normally resistant to glutamate toxicity are made sensitive by elevated extracellular $\mathrm{Ca}^{2+}$ : Toxicity is blocked by the $N$-methylD-aspartate antagonist MK-801. Proc. Natl. Acad. Sci. USA 85:65566560 .

Hajos, F., G. Garthwaite, and J. Garthwaite (1986) Reversible and irreversible neuronal damage caused by excitatory amino acid analogues in rat cerebellar slices. Neuroscience 18: 417-436.

Hamill, O. P., A. Marty, E. Neher, et al. (1981) Improved patch-clamp techniques for high resolution current recordings from cells and cellfree membrane patches. Pflügers Arch. 391: 85-100.

Honoré, T., S. N. Davies, J. Drejer, G. J. Fletcher, P. Jacobsen, D. Lodge, and F. E. Nielsen (1988) Quinoxalinediones: Potent competitive non-NMDA glutamate receptor antagonists. Science 241: 701 703.

Huettner, J. E., and R. W. Baughman (1986) Primary culture of identified neurons from the visual cortex of postnatal rats. J. Neurosci. 6: 3044-3060.

Huettner, J. E., and B. P. Bean (1988) Block of $N$-methyl-D-aspartateactivated current by the anticonvulsant MK-801: Selective binding to open channels. Proc. Natl. Acad. Sci. USA 85: 1307-1311.

Jahr, C. E., and C. F. Stevens (1987) Glutamate activates multiple single channel conductances in hippocampal neurons. Nature 325 : $522-525$.

Johnson, J. W., and P. Ascher (1987) Glycine potentiates the NMDA response in cultured mouse brain neurons. Nature 325: 529-531.

Kass, I., and P. Lipton (1986) Calcium and long-term transmission damage following anoxia in dentate gyrus and CA1 regions of the rat hippocampal slice. J. Physiol. 378: 313-334.

Koh, J. Y., and D. W. Choi (1988) Vulnerability of cultured cortical neurons to damage by excitotoxins: Differential susceptibility of neurons containing NADPH-diapharase. J. Neurosci. 8: 2153-2163.

Kudo, Y., and A. Orgura (1986) Glutamate-induced increase in intracellular $\mathrm{Ca}^{2+}$ concentration in isolated hippocampal neurons. $\mathrm{Br}$. J. Pharmacol. 89: 191-198.

Kurth, M. C., J. H. Weiss, and D. W. Choi (1989) Relationship between glutamate-induced ${ }^{45} \mathrm{Ca}^{++}$influx and resultant neuronal injury in cultured cortical neurons. Neurology 39(Suppl. 1): 217.

Lemasters, J. J., J. DiGuiseppi, A. Nieminen, and B. Herman (1987) Blebbing, free $\mathrm{Ca}^{2+}$ and mitochondrial membrane potential preceding cell death in hepatocytes. Nature 325: 78-81.

Mattson, M. P., P. B. Guthrie, B. C. Hayes, and S. B. Kater (1989) Roles for mitotic history in the generation and degeneration of hippocampal neuroarchitecture. J. Neurosci. 9: 1223-1232.

Mayer, M. L., and G. C. Westbrook (1987) The physiology of excitatory amino acids in the vertebrate central nervous system. Prog. Neurobiol. 28: 197-276.

Mayer, M. L., A. B. MacDermott, G. L. Westbrook, S. J. Smith, and J. C. Barker (1987) Agonist- and voltage-gated calcium entry in cultured mouse spinal cord neurons under voltage clamp measured using arsenazo III. J. Neurosci. 7: 3230-3244.

Murphy, S. N., and R. J. Miller (1988) Kainic acid increased intracellular calcium in mouse striatal neurons by two mechanisms. Abstr. Soc. Neurosci. 14: 94.

Murphy, S. N., S. A. Thayer, and R. J. Miller (1987) The effects of excitatory amino acids on intracellular calcium in single mouse striatal neurons in vitro. J. Neurosci. 7: 4145-4158.

Nehls, D. G., A. Kurumaji, C. K. Park, and J. McCulloch (1988) Differential effects of competitive and non-competitive $N$-methyl-Daspartate antagonists on glucose use in the limbic system. Neurosci. Lett. 91: 204-210.

Neuman, R. S., Y. BenAri, M. Gho, and E. Cherubini (1988) Blockade of excitatory synaptic transmission by 6-cyano-7-nitroquinoxaline2,3-dione (CNQX) in the hippocampus in vitro. Neurosci. Lett. 92. 64-68.

Ogura, A., M. Miyamoto, and Y. Kudo. (1988) Neuronal death in vitro: Parallelism between survivability of hippocampal neurones and sustained elevation of cytosolic $\mathrm{Ca}^{2+}$ after exposure to glutamate receptor agonist. Exp. Brain Res. 73: 447-458.

Olverman, H. J., A. W. Jones, and J. C. Watkins (1984) L-Glutamate has higher affinity than other amino acids for [3H]-D-APS binding sites in rat brain membranes. Nature 307: 460-462.

Pocock, J. M., H. M. Murphie, and D. G. Nicholls (1988) Kainic acid inhibits the synaptosomal plasma membrane glutamate carrier and allows glutamate leakage from the cytoplasm but does not affect glutamate exocytosis. J. Neurochem. 50:745-751.

Price, M. T., T. Honoré, M. E. Mueller, J. Labruyere, E. Silverman, and J. W. Olney (1988) CNQX potently and selectively blocks kainate excitotoxicity in the chick embryo retina. Abstr. Soc. Neurosci. 14(1): 418 .

Pumain, R., I. Kurcewicz, and J. Louvel (1987) Ionic changes induced through excitatory amino acids in the rat cerebral cortex. Can. J. Physiol. Pharmacol. 65: 1067-1077.

Rothman, S. M. (1985) The neurotoxicity of excitatory amino acids is produced by passive chloride influx. J. Neurosci. 5: 1483-1489.

Rothman, S. M., and J. W. Olney (1986) Glutamate and the pathophysiology of hypoxic/ischemic brain damage. Ann. Neurol. 19: 105111.

Rothman, S. M., J. G. Thurston, and R. E. Hauhart (1987) Delayed neurotoxicity of excitatory amino acids in vitro. Neuroscience 22 . $471-480$.

Schanne, F. A., A. Kane, E. Young, and J. Farber (1979) Calcium dependence of toxic cell death: A final common pathway. Science 206: $699-700$.

Siesjö, B. K. (1988) Historical overview: Calcium, ischemia, and death of brain cells. Ann. NY Acad. Sci. 522: 638-661.

Simon, R. P., T. Griffiths, M. C. Evans, J. H. Swan, and B. S. Meldrum (1984) Calcium overload in selectively vulnerable neurons of the hippocampus during and after ischemia: An electron microscopy study in the rat. J. Cereb. Blood Flow Metabol. 4: 350-361.

Stodieck, L. S., and J. J. Miller (1987) Direct evidence for an increase 
in intracellular free calcium in amino acid neurotoxicity of selectively vulnerable hippocampal neurons. Abstr. Soc. Neurosci. 13: 9.

Van Harreveld, A. (1959) Compounds in brain extracts causing spreading depression of cerebral cortical activity and contraction of crustacean muscle. J. Neurochem. 3: 300-315.

Weber, M. L., A. W. Probert, P. A. Boxer, and F. W. Marcoux (1988) The effects of ion channel modulators on hypoxia-induced calcium accumulation and injury in cortical neuronal cultures. Abstr. Soc. Neurosci. 14: 1117.
Wong, E. H. F., J. A. Kemp, T. Priestly, A. R. Knight, G. N. Woodruff, and L. L. Iversen (1986) The anticonvulsant MK-801 is a potent $N$-methyl-D-aspartate antagonist. Proc. Natl. Acad. Sci. USA 83: $7104-$ 7108.

Yamada, K. A., J. M. Dubinsky, and S. M. Rothman (1989) Quantitative physiological characterization of a quinoxalinedione nonNMDA receptor antagonist. J. Neurosci. 9: 3230-3236. 